\title{
Research on MPPT Based on Fuzzy Auto-disturbance Rejection
}

\author{
Ma Chuang, Li Hong, Gong Zhenyu \\ Beijing Information Science \& Technology University Beijing 100192, China
}

\begin{abstract}
For the classical maximum power point tracking algorithm, the setting of small step increases the speed of maximum power tracking of photovoltaic power generation system and the stability of operation in stable state. To solve this problem, this paper designs a maximum power point tracking system based on booster circuit, and adopts fuzzy auto-disturbance rejection control to improve the conductance increment method with variable step length. Based on the relationship between the rate of conductance change and the maximum power point conductance, the step size of the adrc is adjusted by using the fuzzy logic control. The MPPT system of photovoltaic power generation is designed by combining the incremental conductance method and the improved fuzzy control method with the correction factor. The control method does not depend on the mathematical model of the system, the design is flexible, the control effect is robust, stable and accurate. It can be used for reference for other types of solar photovoltaic power generation control system.
\end{abstract}

\section{Introduction}

Solar energy because of its potential in clean long features more and more get people's attention and recognition, photovoltaic cells as taking solar energy as the main energy products, with the traditional battery don't have clean lasting advantages. However, due to the photovoltaic panels acquisition and conversion capacity constraints, can photoelectric conversion efficiency of photovoltaic cells only less than $20 \%$ [1]. The output power varies with the intensity of light and the ambient temperature, and the nonlinear characteristics of power and voltage have their own maximum working point. Therefore, maximum power point tracking technology has become an indispensable content in photovoltaic systems for maintaining the maximum output power of photovoltaic cells.

In recent years, MPPT control method has become a research hotspot [2], such as disturbance observation method [3] conductance increment method [4], particle swarm optimization algorithm [5], fuzzy control [6], neural network algorithm [7]. Disturbance observation affected by disturbance step length, perturbation step size is small, tracking speed slow perturbation step size is larger, after reach the maximum power point larger oscillation, causes the power loss conductance increment method has fast tracking speed faster, but more complex and intelligent algorithm of sensor accuracy requirement high has a higher requirement to the controller, operation more, implementation is more complicated. Adrc [8] is a control algorithm to deal with disturbances. It is a nonlinear control method based on error feedback. It does not rely on the accurate mathematical model of the system, and it can make better estimation and compensation for the unmodeled dynamics and unknown disturbances of the system [9]. In this paper, an improved adrc is designed to simplify the structure of typical adrc, reduce the parameters to be adjusted, and introduce fuzzy control [10] to optimize the parameters of adrc, so that the controller has stronger selfadaptability. An improved auto-disturbance rejection controller was applied to the variable-step conductance increment method to improve the response speed and steady-state accuracy of the system. Simulation results show that the proposed method can effectively improve the dynamic response and power oscillation of photovoltaic system.

\section{Improved incremental conductance method based on ADRC Mathematical model of ADRC}

The ADRC controller is mainly consists of three parts: tracking differentiator (TD), extended state observer (ESO), nonlinear state error feedback (NLSEF) [8]. Fig.1 shows the block diagram of first order standard ADRC.

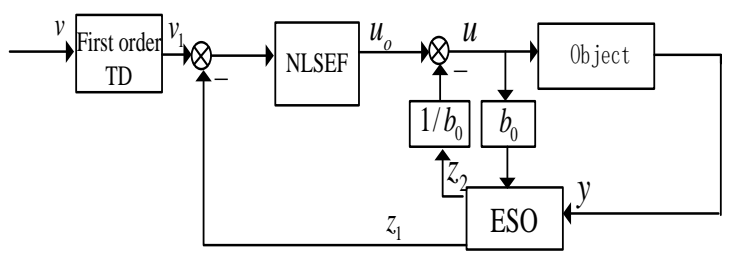

Fig. 1. The block diagram of first order ADRC

In Fig. $1, v$ is the input signal of ADRC controller, $v_{1}$ is the tracking signal of input signal; $y$ is the feedback signal of the system; ${ }^{z_{1}}$ is the tracking signal; $z_{2}$ is the

\footnotetext{
* Corresponding author: Ma Chuang 1064846438@qq.com
} 
total disturbance observation; $b_{0}$ is the compensation factor; $z_{2} / b_{0}$ is the compensation for internal and external disturbances; $u_{0}$ is the initial signal of the controlled object by NLSEF; $u$ is the final control signal after compensating the disturbance.

In engineering applications, the first-order ADRC controller of NLSEF error gain coefficient of $\beta$ is not easy to adjust, under the condition of variable disturbance conditions need to manually adjust the parameters, this is not conducive to the practical application so this article will be used in the design of ADRC controller, fuzzy control using fuzzy rules of NLSEF parameters setting, achieve the goal of online modification parameters, is advantageous to the ADRC controller in the practical engineering application.

\subsection{Fuzzy Automatic Disturbance Rejection Control}

In the control system, the first to know the mathematical model of controlled object or the transfer function, and some complex production process, because the practice is affected by many factors, accurate mathematical model and analysis are hard to obtain, this is the limitation of the classical control theory. According to people's experience, for example, temperature and other parameters can be to judge these complex production system that according to the experience of people judge summed up a lot of kinds of control rules were obtained, by a fuzzy controller is equivalent to the human brain to complete the complex nonlinear system control, it does not rely on mathematical model also can get good control effect.This is fuzzy control.

The input of the fuzzy controller is the error e and the error rate $\mathrm{e}_{\mathrm{c}}$ between the state estimation of the feedback velocity and the given velocity of the system. The output is the modified value $\Delta \beta$ of the parameter to be adjusted in NLSEF. In their domain, 7 language subsets are defined, namely $\{\mathrm{NB}, \mathrm{NM}, \mathrm{NS}, \mathrm{O}, \mathrm{PS}, \mathrm{PM}, \mathrm{PB}\}$, respectively. Where $\mathrm{NB}, \mathrm{NM}, \mathrm{NS}, \mathrm{O}, \mathrm{PS}, \mathrm{PM}$ and $\mathrm{PB}$ are linguistic values. They respectively represent "negative big", "negative medium", "negative small", "0", "positive big", "positive small", "positive medium", "positive big". Mamdani algorithm is used for fuzzy reasoning, and average weighting method is used for defuzzization. The fuzzy rules for setting $\Delta \beta$ are shown in Tab.1.

Tab 1. Fuzzy rule table of $\triangle \beta$

\begin{tabular}{cccccccc}
\hline & \multicolumn{7}{c}{$\mathrm{E}_{\mathrm{C}}$} \\
\hline E & NB & NM & NS & Z & PS & PM & PB \\
NB & PB & PB & PM & PM & PS & Z & Z \\
NM & PB & PB & PM & PS & PS & Z & NS \\
NS & PM & PM & PM & PS & Z & NS & NS \\
Z & PM & PM & PS & Z & NS & NS & NM \\
PS & PS & PS & Z & NS & NS & NM & NM \\
PM & PS & Z & NS & NM & NM & NM & NB \\
PB & Z & Z & NM & NM & NB & NB & NB \\
\hline & & & & & & &
\end{tabular}

After the defuzzification, substituting value $\Delta \beta$ in (1).

$$
\beta=\beta^{\prime}+\Delta \beta
$$

Where ${ }^{\beta^{\prime}}$ is the initial value of error gain in NLSEF.

\section{Improved incremental conductance method}

\subsection{Conductance Increment Method}

In practical application, the photovoltaic array is composed of many series and parallel photovoltaic cells, and the equivalent circuit of photovoltaic cells is shown in Fig. 2:

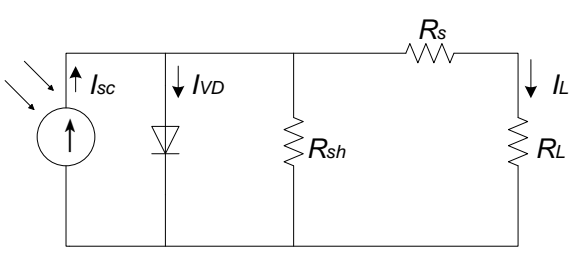

Fig. 2. Equivalent circuit of solar photovoltaic cell

In Fig.2,RL is the external load of photovoltaic cells, the load current is IL,Rs and Rsh is the internal resistance of photovoltaic cells, which is usually small, Rsh is the bypass resistance, and the general resistance is large. IVD is the total diffusion current Isc through p-n junction, which represents the current excited by photons in photovoltaic cells, depending on the irradiance cell area and temperature $\mathrm{T}$.

According to the characteristics of photovoltaic cells, there is only one maximum power point under certain lighting and temperature conditions. During the operation of photovoltaic power generation system, certain control means must be adopted to ensure that photovoltaic panels work near the maximum power point in different environments, so as to improve the conversion efficiency of electric energy. This process is the MPPT control. Incremental conductance method is one of the most commonly used MPPT algorithms. Voltage power curve of the photovoltaic array is a single-peak curve, the maximum output power point, the power on voltage derivative is zero, for maximum power point, as long as in the region of the power of the voltage of the derivative is greater than zero increase the voltage, the power of the derivative of less than zero voltage area decrease the voltage, the derivative is equal to zero or very close to zero, voltage remains unchanged; When the voltage remains unchanged and the current increases, the working voltage increases, and when the voltage remains unchanged and the current decreases, the working voltage decreases. So available:

$\frac{d P_{P V}}{d U_{P V}}=I_{P V} \frac{d U_{P V}}{d U_{P V}}+U_{P V} \frac{d I_{P V}}{d U_{P V}}=I_{P V}+U_{P V} \frac{d I_{P V}}{d U_{P V}}=0$ 
Where, $\mathrm{P}_{\mathrm{PV}}, \mathrm{U}_{\mathrm{PV}}$ and $\mathrm{I}_{\mathrm{PV}}$ are respectively the sampling values of output voltage of photovoltaic cells and output current of photovoltaic cells at time $t ; \mathrm{D}_{\mathrm{PPV}}, \mathrm{dU}_{\mathrm{PV}}$, and $\mathrm{dI}_{\mathrm{PV}}$ are the changes of the output power of photovoltaic cells at time $\mathrm{t}-1$ and time $t$ respectively. In the process of maximum power point tracking, photovoltaic cells first determine whether the system is located at the maximum power point by comparing whether the conductance variation $\mathrm{dI} / \mathrm{dU}$ of the battery and the negative conductance $\mathrm{I} / \mathrm{U}$ of the battery are equal. When they are not equal, the photovoltaic system can determine the position relationship between the output power and the maximum output power at the current moment through the relative size relationship between them, and apply the corresponding disturbance voltage to make the output power approach the maximum power point. Its control strategy flow chart is shown in Fig 3.

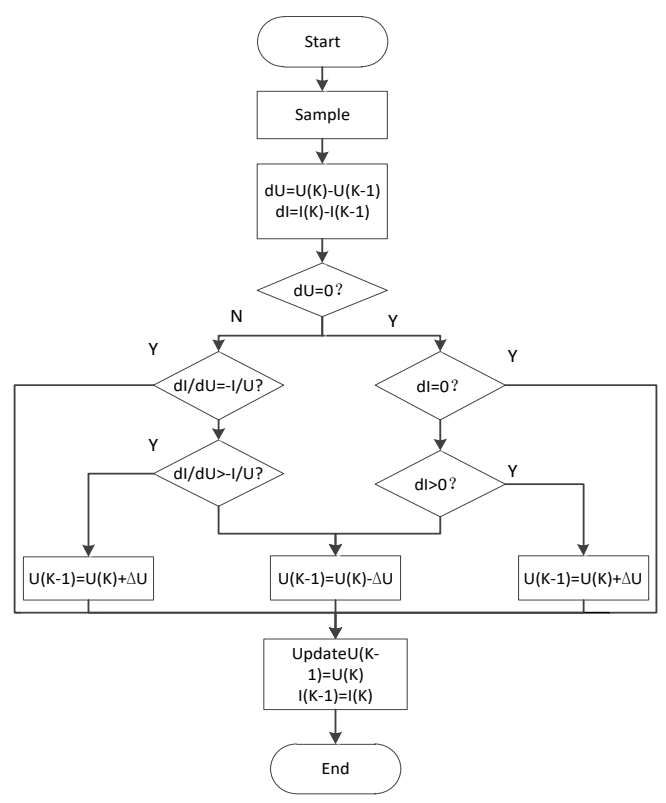

Fig. 3. Routine algorithm program flow chart

Although this method has the capability of dynamic tracking, it still has some defects, such as the contradiction between tracking speed and accuracy and the misjudgment of step direction.

\subsection{Incremental Conductance Method Based on ADRC}

Aiming at the shortcomings of the traditional fixed-step incremental conductance method, and in order to improve the dynamic and steady characteristics of the incremental conductance method in MPPT tracking, the auto-disturbance rejection algorithm is adopted to improve the incremental conductance method. In general, MPPT with constant step size cannot give consideration to both the dynamic response and the reduction of steady state oscillation, and cannot achieve the optimization of both. Here, auto-disturbance rejection algorithm is adopted to realize the change of step size, so as to give consideration to the tracking accuracy and speed.
Let $\mathrm{U}(\mathrm{k})$ and $\mathrm{I}(\mathrm{k})$ be the voltage and current output by photovoltaic cells at moment $\mathrm{k}$, and $\mathrm{D}(\mathrm{k})$ and Step are the changes of duty cycle and duty cycle respectively, namely, the change of step length is:

$$
D(k)=D(k-1) \pm \text { step }=D(k-1) \pm N\left|\frac{d P}{d U}\right|
$$

Here, the increment of conductance should be a constant value of 0 , and the first-order adrc can meet this requirement. The adrc can be designed with the sum of the system conductance increment and instantaneous conductance as input, and the step length change $\mathrm{D}(\mathrm{k})$ as output. Due to differential GPS device is mainly used in the system arrangement of the transition process, and extract the differential signal, but for the first order since immunity regulator, extended state observer and observation of the disturbance of tracking signal output system, only not differential output signal of control object, so the differential tracker only play the role of the filter in the system, to reduce the complexity of model and reduce for setting parameters, differential tracker can be omitted. Because the fal function in nonlinear state error feedback characteristic curve is not smooth, this is not smooth features easy to make the system chattering in after entering the steady state, the linear error control is adopted, which in a simple first-order system with appropriate proportion gain instead of NLSEF module, facilitate the realization of the digital calculation, at the same time to speed up the response of the system. At this point, the first-order ADRC regulator mathematical model is:

The ESO shown as follows:

$$
\left\{\begin{array}{l}
e=z_{1}-e_{0} \\
\dot{z}_{1}=z_{2}-\beta_{01} f a l(e, a, \delta)+b D(t) \\
\dot{z}_{2}=-\beta_{02} f a l(e, a, \delta)
\end{array}\right.
$$

The NLSEF shown as follows:

$$
\left\{\begin{array}{l}
e_{1}=e_{0}^{*}-z_{1} \\
D_{0}(t)=k_{p} e_{1} \\
D(t)=D_{0}(t)-z_{2} / b
\end{array}\right.
$$

In equations (4) and (5) :el is the tracking error of the sum of the system conductance increment and instantaneous conductance; GZ is the sum of the actual conductance increment and instantaneous conductance of the system; $G_{Z}^{*}$ is the system's given value of $0 ; Z 1$ is the state estimation of the sum of the system conductance increment and instantaneous conductance; $\mathrm{Z} 2$ is the observed value of disturbance signal; $f a l(e, a, \delta)$ is the optimal control function, fuzzy rules are used to set parameters in NLSEF. The simplified first-order auto-disturbance rejection regulator block diagram is shown in Fig. 4. 


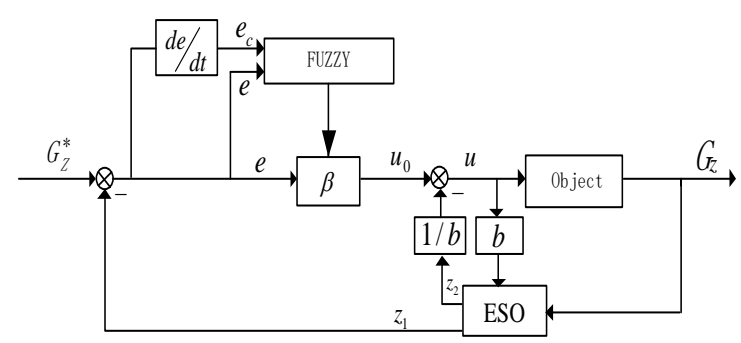

Fig.4 The block diagram of improved first order ADRC speed regulator

\section{Simulation Experiment}

Experimental conditions: the photovoltaic system simulation experimental model shown in Fig. 5 is built by Matlab/Simulink platform:

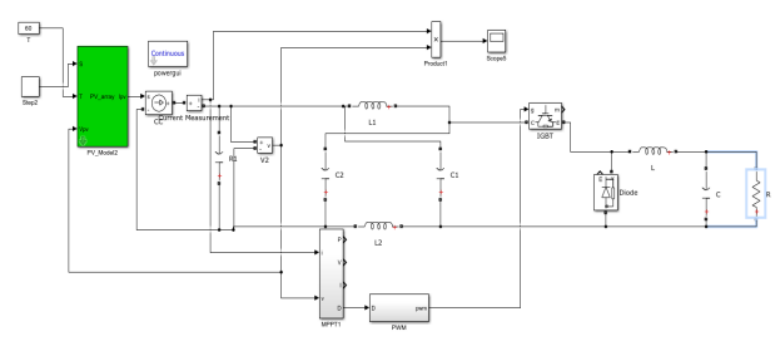

Fig.5 The simulation model

In fig.5, Boost circuit is adopted as the main circuit. The MPPT module collects the voltage and current of photovoltaic panels, generates the duty ratio by adopting the improved conductance increment method based on fuzzy ADRC, converts the obtained results into PWM wave, controls the on-off of switch tube, and realizes MPPT control. Circuit parameters in the simulation experiment: photovoltaic array parallel capacitor $\mathrm{C} 1=1000 \mu \mathrm{F} ; \mathrm{C} 2=300 \mu \mathrm{F} ; \mathrm{L}=0.3 \mathrm{mH}$, initial value of current $\mathrm{IL}=0 \mathrm{~A} ; \mathrm{R}=20 \Omega$; Switch frequency is $20 \mathrm{kHz}$. PV battery array parameters: the short-circuit current $\mathrm{ISC}=13.88 \mathrm{~A}$, open circuit voltage $\mathrm{VOC}=354 \mathrm{~V}$ under standard test conditions $\mathrm{S}=1000 \mathrm{~W} / \mathrm{m} 2, \mathrm{~T}=25$, and the maximum nominal power of PV battery array is about $4.2 \mathrm{kw}$. Based on the traditional incremental conductance method and the improved incremental conductance method based on fuzzy control ADRC, the maximum power point tracking characteristics and steady state power chattering are tested.

In order to verify the control quality of the fuzzy ADRC method for the change of environmental conditions, the abrupt change of the ambient temperature and light intensity is simulated and compared with the traditional method.

The ambient temperature $\mathrm{T}$ remains unchanged, and the illumination intensity S mutation simulation Fig.6 is the simulation waveform of traditional method and ADRC method respectively, $\mathrm{T}=25, \mathrm{~S}$ at $0.15 \mathrm{~s}, 0.25 \mathrm{~s}$ from $1100 \mathrm{~W} / \mathrm{m} 2$ to $900 \mathrm{~W} / \mathrm{m} 2$, and then to $1100 \mathrm{~W} / \mathrm{m} 2$. Fig.6(a) and (b) respectively show the output power waveform of PV cell under the condition of light change.
It can be seen that the output power of PV cell is stable and fluctuates in the transition stage when the external environment changes, the output power transition of Boost circuit adjusted by fuzzy ADRC method is smooth and stable without fluctuation. Fig. 7(c) and (d) show the output current of PV cell. It can be seen that the current of traditional method fluctuates greatly.

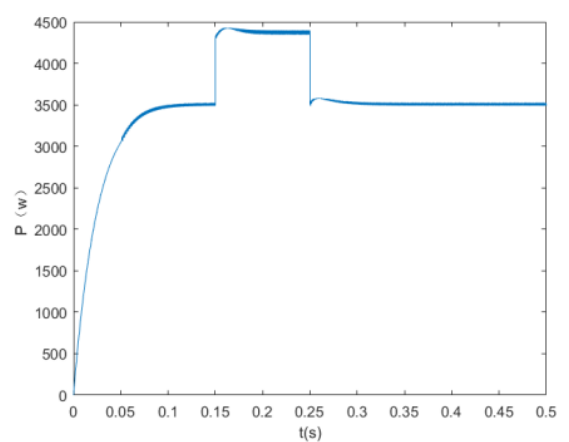

(a)

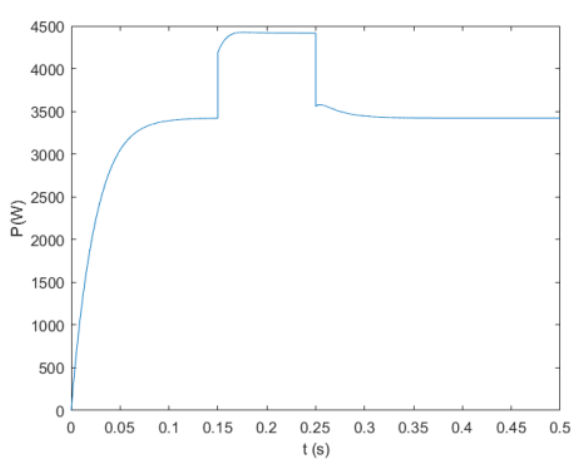

(b)

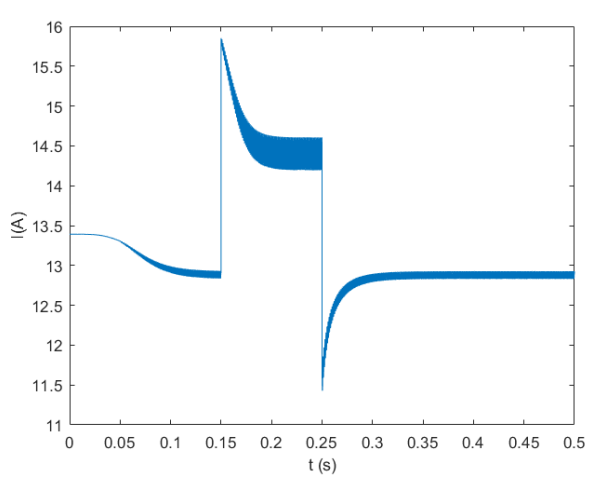

(c) 


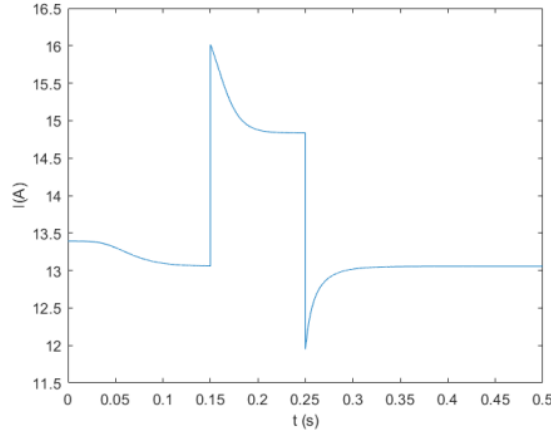

(d)

Fig.6 Simulation waveform comparison

\section{Conclusion}

In this paper, the maximum power point tracking model is established in Matlab/simulink environment by using the auto-disturbance rejection conductance increment method of fuzzy control. On the basis of inheriting the traditional fixed-step conductance increment method, using the idea of adjusting step size, the auto-disturbance rejection controller adjusts step size according to the relationship between the rate of conductance change and the maximum power point conductance. Fuzzy control is used to simplify the process of parameter adjustment in adrc. So that the photovoltaic power generation system can adjust the step size in time according to the change of the environmental state, and effectively balance the contradiction between the response speed and stability of the system. In order to verify the effectiveness and superiority of this fuzzy control algorithm, the simulation and experimental comparison of the new algorithm and the traditional algorithm are carried out on the experimental equipment.

The experimental results show that the ADRC method proposed in this paper has the advantages of fast response, no overshoot and strong robustness, and is superior to the traditional conductance increment method in both rapidity and stability, which can control the MPPT of the output power of photovoltaic cell array.

\section{References}

1. Lin W M, Hong C M, Chen C H. Neural-networkbased MPPT control of a stand - alone hybrid power generation system[J]. IEEE Transactions on Power Electronics, 2011, 26( 12):3571 - 3581 .

2. Rong Desheng,Liu Feng, Research on Improved Disturbance Observation Method in PV MPPT.[J]. Proceedings of the CSU-EPSA.2017,29 (3) :104-109.

3. Yie-Tone Chen, Zhi-Hao Lai, Ruey-Hsun Liang. A novel auto-scaling variable step-size MPPT method for a PV system [J]. Sol Energy ,2014: 247256.

4. Vanxay Phimmasone, Yuta Kondo, Natsuki Shiota, The Effectiveness Evaluation of the newly Improved PSO-based MPPT Controlling Multiple PV Arrays[J]. IEEE Department of Engineering and applied Science, 2013:81-86.

5. Vanay P, Tsugio E, Yuta K, et al. Improvement of the Maximum Power Point Tracker for Photovoltaic Generators with Particle Swarm Optimization Technique by Adding Repulsive Force among Agents[C]//The 12th International Conference on Electrical Machines an Systems Tokyo,Japan,2009:211-217.

6. Khateb A, Rahim N, Selvaraj J.Fuzzy logic controller for MPPT SEPIC converter and PV single-phase inverter[J] IEEE Symp. ISIEA,2011, 25(2): 182-187.

7. Bahgat, A, Helwa, N, Ahmad, G. Maximum power point tracking controller for PV systems using neural networks [J]. Renewable Energy,2005.30(8): 1257-1268.

8. Jingqing Han, Active Disturbance Rejection Control Technique. Beijing: National Defend Industry Press, 2008.

9. Jingqing Han, From PID technique to active disturbance rejection control technique. Control Engineer technique, Vol.3, pp.13-18, May 2002.

10. Wen $\mathrm{Gu}$, Jiuhe Wang, "Vector control strategy of permanent magnet synchronous motor based on auto disturbance rejection control," Journal of Power Supply, vol. 9, pp.65-68, September 2011. 